\title{
Simulation of thermal strength of the process of flat grinding of workpieces in intermittent circles
}

\author{
Nikolai Vetkasov ${ }^{1, *}$, , Sergey Ulitin ${ }^{1}$, Oleg Krupennikov ${ }^{1}$, Valeriy Sapunov ${ }^{1}$, and Andrey \\ Ankilov ${ }^{1}$ \\ ${ }^{1}$ Ulyanovsk State Technical University, Machine building faculty, 32 Severnyy Venets st, Ulyanovsk, \\ 432027, Russia
}

\begin{abstract}
The present article is concerned with numerical schemes of the radial $P_{y}$ and the tangential $P_{z}$ components of the grinding pressure and with the average contact temperature $T,{ }^{\circ} \mathrm{C}$. These characteristics give an opportunity to assess the level of the thermal power strain at every path of the operational cycle of the flat pendulum grinding using a standard grinding wheel (SW), an intermittent grinding wheel (IGW) and a composite grinding wheel (CGW). Value forecasting of forces $P_{y}, P_{z}$ and the temperature $T$ will make it possible to manage the grinding process and will give the opportunity to avoid the defects precipitation at the grinding finish of the workpiece.
\end{abstract}

\section{Introduction}

Saturation with interrelated automated systems is a key characteristic of the modern machine-building industry. Stable functioning of these systems is provided with the trouble-free operation of all their minor components during the design service life. Providing of the dependable and trouble-free operation of machines and mechanisms suggests the continuous improvement of manufacturing technologies. This is accompanied by the increase in the efficiency of the mechanical processing of workpieces, the severization of requirements to the manufacturing quality (roughness, surface-to-surface accuracy, structure of the surface layer material and etc.). In addition to the above the developed technologies shall be environmental responsible and resource-saving. Depicted tendencies are up-to-date and important for all types of mechanical processing including but not limited to grinding which is the most commonly used finishing method of workpieces especially made of difficult-to-machine materials (heat-treated alloys, ceramics and etc.). The motive to increase the grinding productivity and simultaneously to provide the required quality of grinded workpieces often leads to the fact that burns and tiny fractures appear at the grinding finish of the workpiece. Application of the abrasive tool with the intermittent working surface is a promising trend in the grinding field which can help to resolve this issue. This trend gives the opportunity to manage the temperature in the

\footnotetext{
*Corresponding author: nppwt@ulstu.ru
} 
grinding zone. Intermittent and composite grinding wheels inter alia can be used as the abrasive tool with the intermittent working surface. Because of their nature and structure, they are more complicated tools in terms of manufacturing and application in comparison with SW.

Shchepochkina V.A. [4], Korshunova D.A. [5] and etc. devoted papers to studying the thermal power strain of grinding using CGW and IGW. S. Shei and V. Radkhakrishnan studied various compositions of solid lubricants, and also the opportunity and the technological efficiency of their application with grinding operations instead of cooling and lubrication technological fluids (CLTF) [12].

However, the known mathematical models in some circumstances do not consider such features of grinding using CGW as the opportunity of the fact that FCM will penetrate and implement their functions in the cutting zone, physical and chemical properties of FCM, its damping action and etc. In this context, issues of using of these wheels shall undergo the background study. Mathematical models of thermal power strain during the flat pendulum grinding operation cycle using SW, IGW, CGW contained in this paper, give the opportunity to assess the influence of the radial $P_{y}$ and the tangential $P_{z}$ components of the grinding pressure, and also the average contact temperature $T$.

However, the known mathematical models consider not all features of grinding using CGW, for example, the opportunity of the fact that FCM will penetrate and implement their functions in the cutting zone, physical and chemical properties of FCM, its damping action and etc. As can be seen from the above, the purpose of this paper is to resolve the issue of the mathematical simulation of thermal physics of the intermittent grinding process and inter alia the flat grinding using the CGW.

The development objective of the mathematical model system of the thermal power strain of the flat pendulum grinding cycle by the periphery of SW, IGW, CGW was considered in detail taking into account features of the structure and the grinding process of every studied type of abrasive wheels. It is worth noting that the management efficiency of the grinding process can be materially improved by implementing to the machinery production the relevant scientific functional in the form of adequate mathematical models. These mathematical models give the opportunity to identify output parameters $\left(P_{y}, P_{z}\right.$ forces and the average contact temperature $T$ ) with input parameters: the grinding mode, physical and chemical properties of the workpiece material and FCM of lubrication elements (LE) of CGW, the typical size of the grinding wheel (GW), number and geometrical dimensions of CE of IGW and CGW, the grain size and the bond of GW, cooling method and etc. Studied output parameters $\left(P_{y}, P_{z}, T\right)$ commonly influence the quality of the future workpiece. Therefore, their forecasting during the mathematical simulation when designing the grinding operation will give the opportunity to avoid the appearance of grinding burns, tiny fractures, structural changes in the surface layer material and will provide that workpieces will have preset parameters with the minimum turnaround time i.e. with the maximum performance.

Main feature of the intermittent grinding, typical of using IGW and CGW, is the presence of additional (force and shock) impacts of the processed surface of the blank. These impacts shall be considered when designing mathematical models describing the grinding process using abrasive wheels with the intermittent working surface.

Authors $[8,9]$ proposed the mathematical model of the radial component of the grinding pressure $P_{y}$ submitted in the form of the sum of the mechanical and the hydrodynamic components:

$$
P_{y}=\left[P_{y m} \cdot\left(1-\frac{S_{s e}}{S_{k}}\right)+P_{y g} \cdot\left(1-\frac{H_{s e}}{H_{k}}\right)\right] \cdot K_{p s},
$$


where $P_{y m}$ is the mechanical component of the radial cutting force $P_{y}, \mathrm{H} ; P_{y g}$ is the hydrodynamic component of the radial cutting force $P_{y}, \mathrm{H} ; H_{s e}$ is the height of LE in the periphery of the wheel, $\mathrm{m} ; H_{k}$ is the height of the solid GW, m; $S_{k}$ is the area of the working surface of the periphery of the solid $\mathrm{GW}, \mathrm{m}^{2} ; S_{s e}$ is the area of the working surface of the periphery of the wheel, occupied by LE, $\mathrm{m}^{2} ; K_{p s}$ is the force transmission coefficient determining the force portion transmitted from GW to the blank.

In the dependence (1) the intermittence of the grinding process is considered by the introduction of components $H_{s e}, S_{s e}$ which determine geometrical parameters of CE of IGW and CGW. In addition to the above, the received model gives the opportunity to calculate the force $P_{y}$ both for SW and for IGW and CGW with radial slots and grooves.

Dependence (1) after series of transformations takes the following form:

$$
\begin{aligned}
P_{y}= & {\left[\frac{\pi \cdot \sigma_{t} \cdot \xi^{\lambda_{p}} \cdot\left(\frac{\rho_{z}^{0,92}}{0,147} \cdot a_{c}^{1,08}+5,2 \cdot \mu_{t r} \cdot a_{c}^{1,54} \cdot \rho_{z}^{0,46}\right) \cdot \frac{L_{\min }}{L_{\max }} \cdot Z}{8} \cdot\left(1-\frac{S_{s e}}{S_{k}}\right)+\right.} \\
& \left.+\frac{\varepsilon \cdot \mu_{\text {sog }} \cdot V_{k} \cdot R_{k} \cdot H_{k} \cdot K_{p r}}{0,1 \cdot d_{0}} \cdot\left(1-\frac{H_{s e}}{H_{k}}\right)\right] \cdot\left(\mu_{d} \cdot \sqrt{1+\left(2 \cdot n \cdot \frac{\omega}{p^{2}}\right)^{2}}\right),
\end{aligned}
$$

where $\xi$ is the cutting shrinkage ratio (when grinding $\xi=1,1-2,0$ ); $\lambda_{p}$ is the contraction polytropic index (when grinding $\lambda_{p}=1,25$ ); $\sigma_{t}$ is the blank material yield strength, $\mathrm{N} / \mathrm{mm}^{2}$; $\rho_{z}$ is the top corner radius of the abrasive grain (AG), mm; $a_{c}$ is the scratch depth made by $\mathrm{AG}, \mathrm{mm} ; \mu_{m p}$ is the coefficient of sliding friction of AG atop the metal in the presence of CLF; $L_{\min }, L_{\max }$ is the minimum and the maximum contact arc length of the single AG with the processed blank surface, $\mathrm{mm}$; $Z$ is the number of AG located within the contact area of GW with the processed blank, pcs; $\varepsilon$ is the empirical coefficient $(\varepsilon=1,5-2,5)$; $\mu_{\text {sog }}$ is the dynamic viscosity of the cooling and lubrication fluid (CLF), $\mathrm{Pa} \cdot \mathrm{s} ; V_{k}$ is the peripheral speed of $\mathrm{GW}, \mathrm{m} / \mathrm{s} ; R_{k}$ is the radius of $\mathrm{GW}, \mathrm{mm} ; K_{p r}$ is the coefficient considering the applicability of the hydrodynamic theory of lubrication to the grinding process $\left(K_{p r}=0,6\right)$; $d_{0}$ is the diameter of grains of the main fraction of $\mathrm{GW}, \mathrm{mm} ; \mu_{d}$ is the dynamic factor; $\omega$ is the cycle vibration frequency of $\mathrm{GW}, \mathrm{s}^{-1} ; p$ is the normal vibration frequency of $\mathrm{GW}, \mathrm{s}^{-1} ; n$ is the damping coefficient, $\mathrm{s}^{-1}$.

Force $P_{y}$ determined by dependencies $(1,2)$ is the maximum value of the full cycle of the flat pendulum grinding by the wheel periphery. Methods for determining of force values $P_{y}$ of every working and sparking-out cycle of GW depending on the required roughness of the grinding finish are considered in papers [9] and [11]. Mathematical models [9 and 11] give the opportunity to monitor changes of the force intensity during the grinding process and forming the roughness of the processed blank surface throughout the duration of the working grinding cycle.

Authors based the mathematical model of the tangential component of the grinding pressure $P_{z}$ on the dependence for grinding SW determined by Unyanin A.N. [10] and during its adaptation to intermittent grinding conditions using IGW and CGW transformed it to the form [8]: 


$$
\begin{aligned}
P_{z}= & {\left[B_{d} \cdot\left(\mu_{0} \cdot \sin \gamma+\cos \gamma\right) \cdot L_{k} \cdot a_{k r} \cdot\left(\frac{a_{k r}^{2}}{3}-f_{i d s r}^{2}\right)+\mu_{t r} \cdot c \cdot \sigma_{t} \cdot D_{d} \cdot L_{k} \cdot a_{k r}+\right.} \\
& \left.+\tau_{s} \cdot B_{p} \cdot D+D_{p} \cdot \tau_{s} \cdot \mu_{0} \cdot\left(f_{i} \cdot L_{k}+\frac{A \cdot L_{k}^{2}}{2}\right)\right] \cdot\left(1-\frac{f_{T S M} \cdot S_{s e}}{S_{k}}\right) \cdot K_{p s},
\end{aligned}
$$

where $f_{T S M}$ is the coefficient, showing the area particle of the blank surface, grinded using CGW, processed and finishing with FCM [7]; $\tau_{s}$ is the shearing stress when microcutting using the blank material grain, $\mathrm{Pa} ; f_{i}$ is the cumulative splitting value of grains during the dressing and the dimensional wear of GW, $\mathrm{m} ; L_{k}$ is the arc contact length of the wheel with the blank, $\mathrm{m} ; \gamma$ is the half-angle apical AG, grad; $c$ is the coefficient $(c=$ 1000); $a_{k r}$ is the micro-cutting critical depth, $m$; $f_{i d s r}$ is the wear of AG during the plastic deformation, $\mathrm{m} ; D, B_{p}, A, D_{p}, B_{d}, D_{d}$ and dependence components determined by Unyanin A.N.

The coefficient $f_{T S M}$ introduced by authors to the dependence (3) after theoretical and experimental studies of FCM penetration and lubrication mechanisms when grinding using CGW gave the opportunity to specify the mathematical model and to approach it to real circumstances of friction processes in the grinding zone [7].

As it is known, the component of the grinding pressure $P_{z}$ materially influences the heat density in the contact zone «GW - blank»:

$$
q_{v y d}=\frac{P_{z} \cdot S_{k t} \cdot 10^{6}}{V_{k}},
$$

where $q_{v y d}$ is the areal density of the heat flux emitted in the contact zone, $\mathrm{J} /\left(\mathrm{mm}^{2} \cdot \mathrm{s}\right) ; S_{k t}$ is the area of contact between the wheel and the blank, $\mathrm{m}^{2}$.

Sizy Yu.A. and Stepanov M.S. connected the heat source (the contact plane between the wheel and the blank) with the Universal Transverse Mercator XYZ with the center in the middle of the source. As the result they determined the formula of the average contact temperature $T$ for the round outer grinding using SW and IGW with radial grooves [13]. In addition to the above, the intermittent grinding represents the variety of heating-cooling cycles when the point at the workpiece surface goes through the contact arch $L_{k}$. It depends on the number of LE and their geometrical dimensions. When authors adapted this formula to flat grinding process conditions by the periphery of IGW and CGW with radial slots or grooves, SW obtained the following dependencies for determining $T$ when grinding using SW, IGW and CGW:

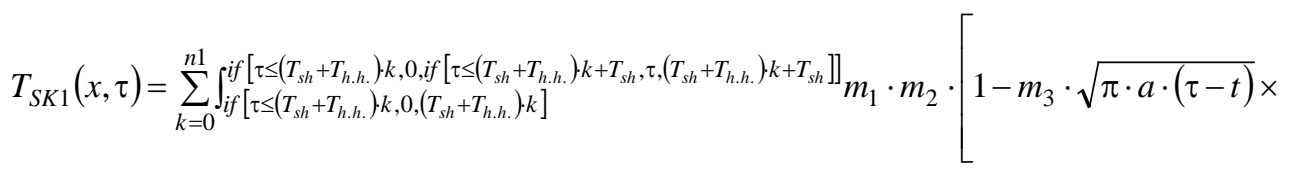

$$
\begin{aligned}
& \left.\left.\times\left[1-e r f\left[\frac{x}{\sqrt{4 \cdot a \cdot(\tau-t)}}+m_{3} \cdot \sqrt{a \cdot(\tau-t)}\right]\right] \cdot e^{\left[\frac{x}{\sqrt{4 \cdot a \cdot(\tau-t)}}+m_{3} \cdot \sqrt{a \cdot(\tau-t)}\right.}\right]^{2}\right] d t
\end{aligned}
$$

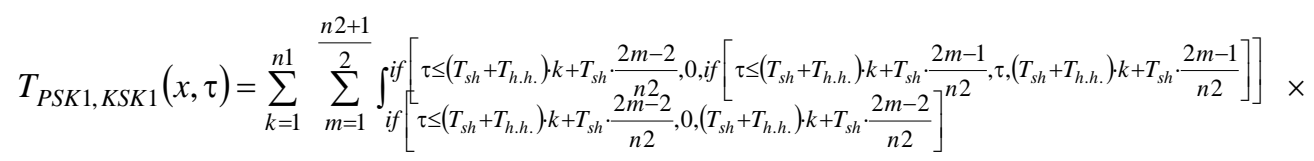




$$
\begin{aligned}
& \times m_{1} \cdot m_{2} \cdot\left[1-m_{4} \cdot \sqrt{\pi \cdot a \cdot(\tau-t)} \cdot\left[1-\operatorname{erf}\left[\frac{x}{\sqrt{4 \cdot a \cdot(\tau-t)}}+m_{4} \cdot \sqrt{a \cdot(\tau-t)}\right]\right] \times\right. \\
& \left.\times e^{\left[\frac{x}{\sqrt{4 \cdot a \cdot(\tau-t)}}+m_{4} \cdot \sqrt{a \cdot(\tau-t)}\right]^{2}}\right] \\
& m_{1}=\frac{i f\left(k<n 1, q 1_{k}, q 1_{n 1}\right) \cdot a}{2 \cdot \lambda} ; m_{2}=\frac{2 \cdot e r f\left[\frac{h}{\sqrt{4 \cdot a \cdot(\tau-t)}}\right]}{\sqrt{\pi \cdot a \cdot(\tau-t)}} \cdot e^{\frac{-x^{2}}{4 \cdot a \cdot(\tau-t)}} \\
& m_{3}=\frac{\text { if }\left[\tau \leq\left(T_{s h}+T_{h . h .}\right) \cdot k+T_{s h}, \alpha 1, \alpha\right]}{\lambda} ; m_{4}=\frac{i f\left[\tau \leq\left(T_{s h}+T_{h . h .}\right) \cdot k+T_{s h} \cdot \frac{2 m}{n 2}, \alpha 1, \alpha\right]}{\lambda} ; \\
& T_{S K, P S K, K S K}(x, \tau)=T_{S K 1, P S K 1, K S K 1}\left[x, \tau+\left(T_{\text {sh }}+T_{h . h .}\right)\right]+T_{0},
\end{aligned}
$$

where $T_{s h}$ is the time of one grinding/sparking-out cycle, $s ; T_{h . h}$. is the total time of free movements of GW between working cycles, $\mathrm{s}$; $k$ is the number of the heating/cooling cycle; $\alpha=0,0018 \mathrm{~J} /\left(\mathrm{mm}^{2} \cdot \mathrm{s} \cdot{ }^{\circ} \mathrm{C}\right)$ is cooling using the dry air; $\alpha_{1}=0,045 \mathrm{~J} /\left(\mathrm{mm}^{2} \cdot \mathrm{s} \cdot{ }^{\circ} \mathrm{C}\right)$ is cooling using CLF (coating); $a$ is the thermal conductivity coefficient, $\mathrm{mm}^{2} / \mathrm{s} ; \lambda$ is the thermal conductivity coefficient of the blank material, $\mathrm{J} /\left(\mathrm{mm} \cdot \mathrm{s} \cdot{ }^{\circ} \mathrm{C}\right) ; \mathrm{x}$ is the temperature measurement depth (distance from the surface), $\mathrm{mm} ; T_{0}$ is the temperature at the beginning of the experiment, ${ }^{\circ} \mathrm{C}\left(T_{0}=20^{\circ} \mathrm{C}\right)$; $n 1$ is the number of contacts between $\mathrm{GW}$ and the blank during the grinding working cycle; $n 2$ is the number of «heatingcooling» semi-cycles of the blank with cutting cusps/cavities of (LE) IGW (CGW).

\section{Results of studies}

Studies were carried out in the form of the mathematical simulation in Microsoft Excel and Mathcad software packages of the flat pendulum grinding working cycle including grinding with infeeding and sparking-out. During the simulation the following input parameters of the grinding process were varied:

- Blank material - steel 5XHB; 40X13; P6M5;

- Speed of the longitudinal feed of the blank $V_{s t}-6 ; 8 ; 12 ; 14 ; 18 \mathrm{~m} / \mathrm{min}$;

- Infeeding $S_{2 x}-0,002 ; 0,004 ; 0,008 \mathrm{~mm} /$ double pass;

- Number of structural elements (slots $z_{p}$ or grooves $z_{p r}$ in IGW; LE $z_{s e}$ of CGW) - 8; 16; 24 pcs;

- Length of structural elements of IGW $\left(L_{p} ; L_{p r}\right)$ and CGW $\left(L_{s e}\right)$ by the periphery - 8; 16; $24 \mathrm{~mm}$.

Other input parameters of the simulation were considered as constant ones for every series of experiments. Results of the computer mathematical simulation of the power strain of the grinding process using SW, IGW and CGW are depicted in Fig. 1. a Results of the computer mathematical simulation of the thermal strain of the grinding process using $\mathrm{SW}$, IGW and CGW are depicted in Fig. 2-3. 
In addition to the above, in Fig. 1 under specific conditions the first 20 cycles correspond to the infeeding grinding, and beginning from the $21^{\text {st }}$ cycle correspond to the sparking-out.

a

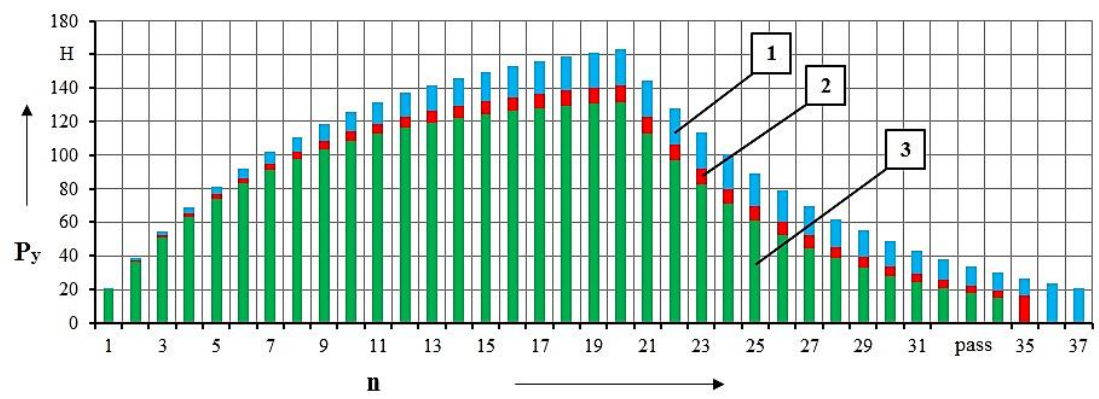

b

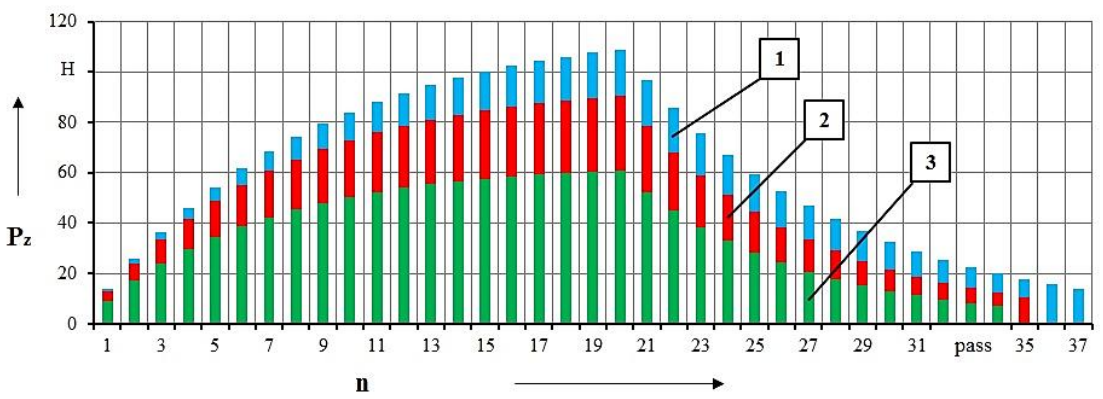

Fig. 1. Radial $P_{y}$ and tangential $P_{z}$ components of the grinding pressure depending on the number of working cycles $n$ : a, b $-P_{y}$ and $P_{z}$ respectively; 1 , 2, 3 - respectively SW, IGW, CGW; wheel $-25 \mathrm{~A}$ F80 K 5 K 35 A 2; number of CE in CGW (IGW) $z_{s e}=16$ pcs; length of in CGW (IGW) by the periphery $L_{s e}=16 \mathrm{~mm}$; blank material - steel 40X13; material of CE in CGW - phenol alkyds + molybdenum disulfide; $V_{s t}=12 \mathrm{~m} / \mathrm{min}$; infeeding $S_{2 x}=0,004 \mathrm{~mm} /$ double pass; required roughness of the processed surface $R a=0,8$ micron

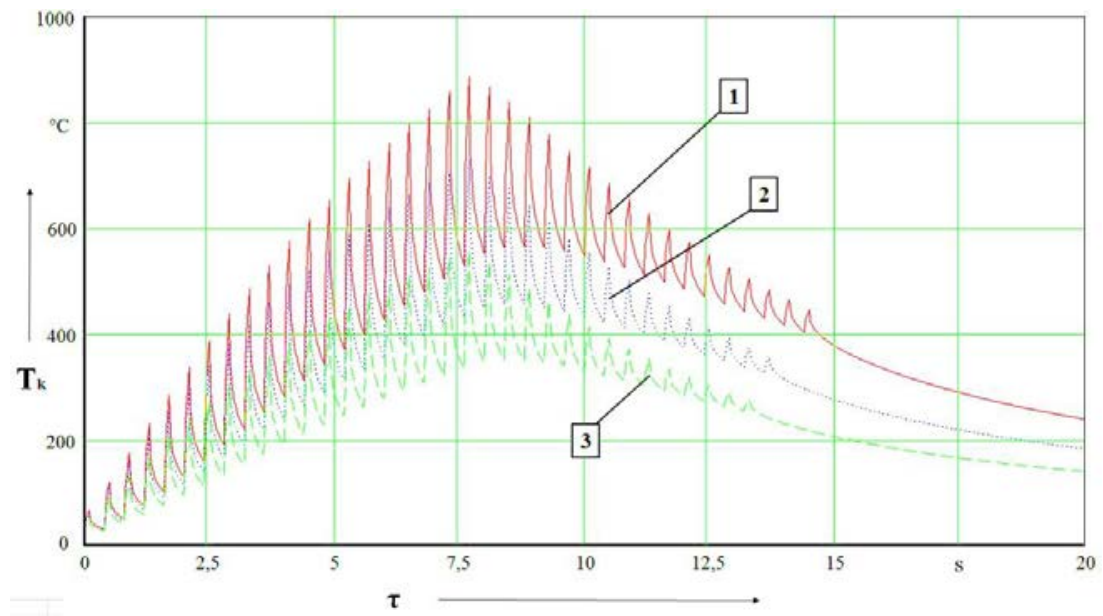

Fig. 2. Average contact temperature $T_{k}$ of the grinding cycle: $1-3-T$ for grinding using SW, IGW, CGW respectively; blank size toward moving of $\mathrm{GW} \mathrm{l}_{\text {zag }}=20 \mathrm{~mm}$; overrun of $\mathrm{GW}_{\text {per }}=10 \mathrm{~mm}$; depth of temperature changing $\mathrm{x}=0 \mathrm{~mm}$ (at the blank surface); cooling - using CLF (coating); other conditions ref. in the legend to Fig. 1 
a

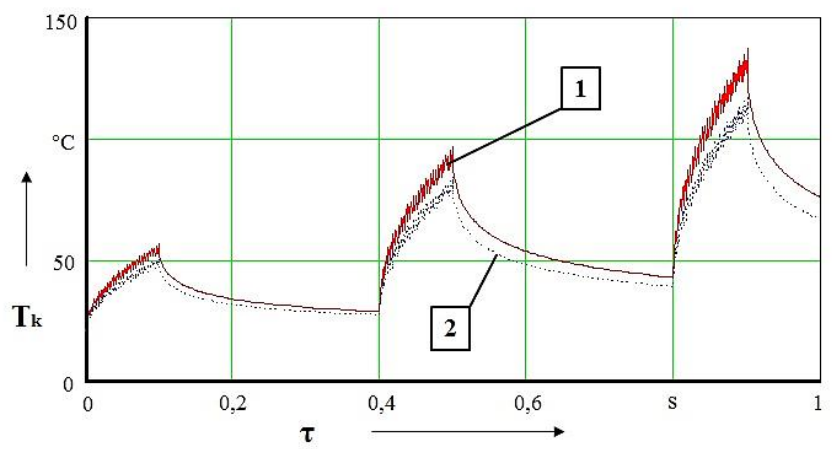

b

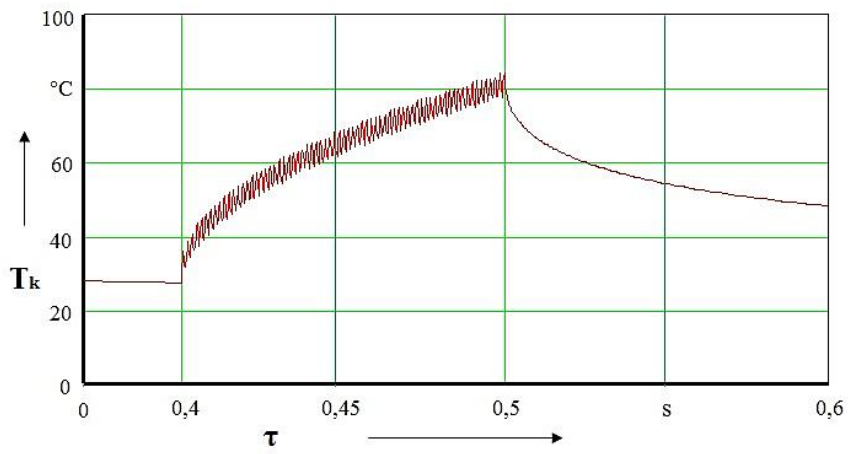

Fig. 3. Changing of the average contact temperature $T_{k}$ : a - at the $1^{\text {st }}-3^{\text {rd }}$ grinding cycles depending on the GW type; b - of single «heating - cooling» cycles at $2^{\text {nd }}$ grinding cycle using CGW; $1 ; 2-T_{k}$ for grinding using IGW and CGW respectively; other conditions ref. in the legend to Fig. 2

Fig. $2-3$ show that models $(5-7)$ give the opportunity to assess the maximum value of the average contact temperature $\mathrm{T}$ of the full working grinding cycle using SW, IGW, CGW at every separate cycle of the wheel, and also to track changing of the temperature at the blank surface (or at the specified depth $x$ ) when interacting with cutting cusps of IGW, cutting cusps and CE in CGW. In addition to the above, it shall be noted that the determination of the heat density level of the grinding cycle will give the opportunity to forecast the appearance of grinding deficiencies at the workpiece surface which is necessary for the guaranteed elimination of tiny fractures and burns at the grinding finish of the workpiece.

According to results of the simulation, the following conclusions were drawn:

1. In the vast majority of cases CGW outperforms SW and IGW in thermal power strain and productivity parameters. However, the different degree of impact of studied simulated parameters on the thermal power strain of the grinding process using CGW and on forming of the blank surface roughness grinded using CGW was identified.

2. It was identified that in case of increase in length of CE in CGW by the periphery $L_{s e}$ from 8 to $24 \mathrm{~mm}$, and the number of CE $z_{s e}$ from 8 to 24 pcs gives the opportunity to decrease in average $P_{y}$ by $9 \%, P_{z}$ and $T$ by $25 \%$ and to increase the productivity of blank processing to the preset roughness $R a$ in average by 3 cycles.

3. It was identified that the application of CGW on the ceramic bond in comparison with CGW on the Bakelite bond provides the decrease of $P_{y}$ in average by $5 \%, P_{z}$ and $T$ by $10 \%$.

4. When grinding blanks of materials of different processability groups, the increase in the labor requirement for processing of blanks of materials from higher processability 
groups was identified. That lead to the increase in $P_{y}, P_{z}, T$ and the increase in number of sparking-out cycles to reach the required roughness by the parameter $R a$. cooling» semicycles of the blank with cutting cusps/cavities of (LE) IGW (CGW).

\section{Conclusion}

As can be seen from the above, the complex of interrelated mathematical models was designed. These models give the opportunity to assess the thermal power strain of the full flat pendulum grinding cycle by the periphery of SW, IGW and CGW. Tested wheels were comparatively assessed by studied output parameters of the grinding process (the radial $P_{y}$ and the tangential $P_{z}$ components of the grinding pressure; the average contact temperature T). Technological recommendations related to engineering and applying of grinding wheels with the rational structure were designed. These recommendations are based on the application of presented models in case of the manufacturing preparation cycles of the blank with cutting cusps/cavities of (LE) IGW (CGW).

\section{References}

1. V.A. Sipaylov, Thermal processes in grinding and the control of surface quality (Moscow, 1978)

2. A.V. Yakimov, Optimization of the grinding process (Moscow, 1975)

3. L.V. Khudobin, N.I. Vetkasov, Grinding the composite wheels (Ulyanovsk, 2004)

4. V.A. Shchepochkin, Development and research of technology of sharpening of cutting tools by composite grinding wheels (Ulyanovsk, 2002)

5. D.A. Korshunov, Development and research of technology of internal grinding by composite wheels (Ulyanovsk, 2001)

6. N.I. Vetkasov, O.G. Krupennikov, S.I. Ulitin, University science in modern conditions: proceedings of the 52nd scientific and technical conference 1, pp. 78-81 (2018)

7. N.I. Vetkasov, O.G. Krupennikov, S.I. Ulitin Innovative technologies in metalworking, pp. 381-386 (2019)

8. N.I. Vetkasov, O.G. Krupennikov, S.I. Ulitin, E.S. Motlyakh, Fundamental and Applied Problems of Engineering and Technology 2 (316), pp. 19-27 (2016)

9. N.I. Vetkasov, O.G. Krupennikov, S.I. Ulitin, International conference on modern trends in manufacturing technologies and equipment (ICMTMTE) 1, pp. 15-22 (2015)

10. A.N. Unyanin, Scientific and technological support for the grinding of workpieces of plastic steels and alloys, preventing clogging of abrasive wheels (Ulyanovsk, 2006)

11. A.I. Armer, Improving the effectiveness flat pendulum grinding through an accelerated nursing with the use of devices for microsupply workpieces (Ulyanovsk, 2002)

12. Komanduri R., Hou Z.B., International journal of Mechanical Sciences, 43-1, 1, pp. 57-88 (2001)

13. Yu.A. Sizyy, M.S. Stepanov, Eastern-European Journal of Enterprise Technologies, 28, 1, pp. 52-63 (2004) 